\title{
A Fiber Coupling Plug for Multi-Object Exoplanet Search Spectral Interferometer
}

\author{
Kai Zhang ${ }^{1,2,3}$, Yongtian Zhu ${ }^{1,3}$, Zhongyu Yue ${ }^{1,3}$ and Lei Wang ${ }^{1,3}$ \\ ${ }^{1}$ National Astronomical Observatories / Nanjing Institute of Astronomical \\ Optics \& Technology, Chinese Academy of Sciences, \\ Nanjing 210042, China \\ ${ }^{2}$ Email: kzhang@niaot.ac.cn \\ ${ }^{3}$ Key Laboratory of Astronomical Optics \& Technology, Nanjing Institute of Astronomical \\ Optics \& Technology, Chinese Academy of Sciences, \\ Nanjing 210042, China
}

\begin{abstract}
The increasing number of spectral survey telescopes that are soon commissioned for exploring the universe will confront us with many new challenges on the use of optical fibers. A fiber-switching problem for telescope's terminal instrument is as important as a well-known problem of positioning fibers on telescope's focal plane. For a modern survey telescope, it generally feeds several terminal instruments with different functions. According to the observation, these instruments are seriously in need of switching fiber-link with telescope as soon as possible. A kind of multi-fiber coupling plugs introduced in this paper will work for switching fiber-link between Multi-object Exoplanet Search Spectral Interferometer (MESSI) and low resolution spectrograph at Guoshoujing telescope (LAMOST). It includes an inserter, a receptor and a socket in general. The number of simultaneously coupling fibers is up to 25 per group, and any broken fiber in a single plug can be replaced by a new standard one soon without wasting entire fiber bundle. In addition, Most of mechanical part can also be individually replaced when a normal loss happens after a long working time. The related test result is given in detail, including the machining precision and coupling efficiency.
\end{abstract}

Keywords. Fiber survey telescope, multi-fiber coupling plug, coupling efficiency

\section{Introduction}

A kind of multi-fiber coupling plugs is commissioned to switch the fiber-link between Multi-object Search Spectral Interferometer (MESSI) and low resolution spectrograph at Guoshoujing telescope (LAMOST) (Kai Zhang et al. 2012). It includes an inserter, a receptor and a socket shown in Figure 1. In this multi-terminal switching scheme, a fiber bunch with identical receptors from telescope is defined as Bus, and the other fiber bunches with identical inserters from different terminals are defined as Extension, in general. The number of simultaneously coupling fibers is up to 25 per group of coupling plugs. The most important technical aspect of these prototypes is that each pair of fibers with ceramic ferrules is spring-loaded to reduce insert/return loss and keep a steading coupling efficiency in the long term. A 3-step aligning method is also adapted to keep every pair of fibers accurately inserted into an independent coupling cavity. The 3 -step alignment shown schematically in Figure 2 includes:

(a) A connecting ring which is similar to the one in multiple fiber push-on/pull-off type (MPO), 

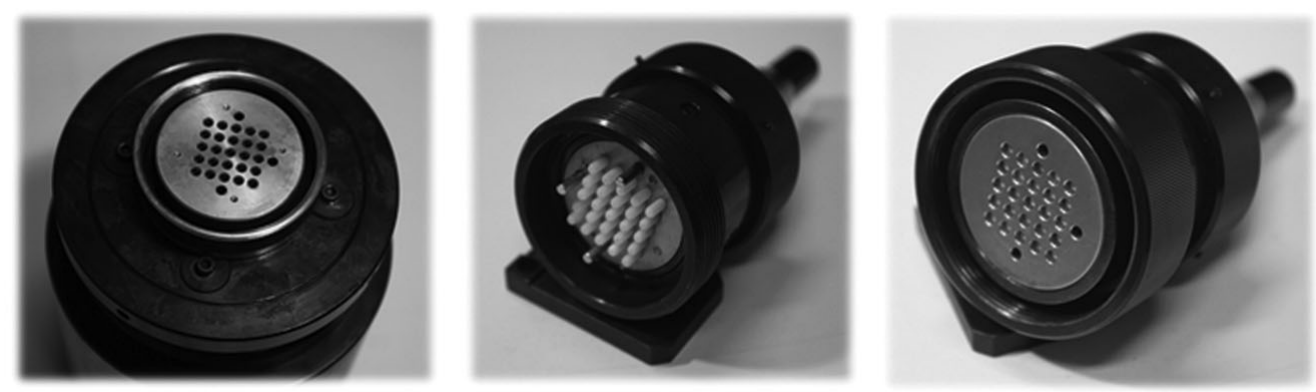

Figure 1. Multi-fiber coupling plugs (left: Socket; Mid: Inserter; Right: Receptor)

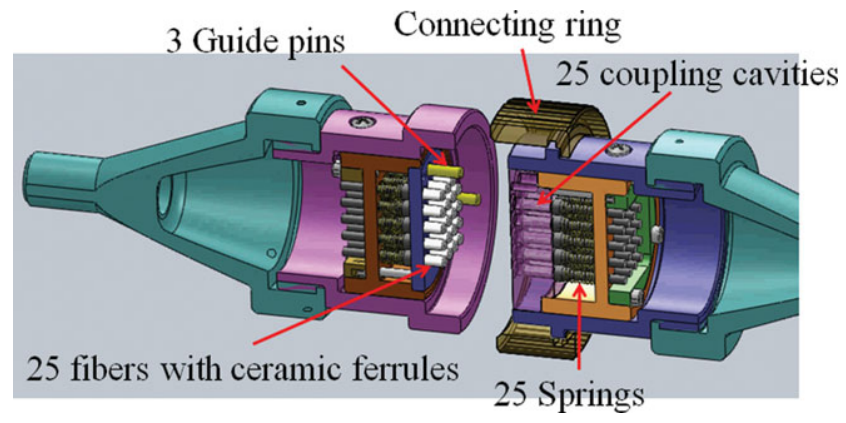

Figure 2. 3-step alignment configuration

(b) Three guide pins that give general alignment with the precision of $<0.10 \mathrm{~mm}$, and

(c) Twenty five coupling cavities each of which giving an adaptive aligning precision of $5 \mathrm{um}$.

\section{Test Result}

The commercial ceramic ferrules without an ideal concentricity and multi-mode optical fibers of 320 micron in diameter are used in the prototype development. The following items of test result fully illustrate the current performance of prototypes manufactured by the traditional high-precision machining:

(a) A guiding plane with 25 coupling cavities is the most important part in the plug design. The positioning and aperture accuracy of 25 drilling holes are smaller than 10 micron, which determines the final coupling efficiency (Fig. 3).

(b) Beam quality after coupling between an inserter and a receptor usually gets worse due to the inner propagation mode of fiber. It is easily changed when fibers are coupled or strongly bent. The Gaussian-like flux curve in Figure 4 proves a good performance in this aspect (Note that different max flux in the figure is caused by the different input intensity in test).

(c) Multi-fiber coupling efficiency is the point in this research. The average value of coupling efficiency is about $83 \%$ in this test (Fig. 5).

\section{Conclusions}

Although the ideal performance was not really achieved, the above result still gives us certain confidence in this research. More improvement needs to be done in the next 

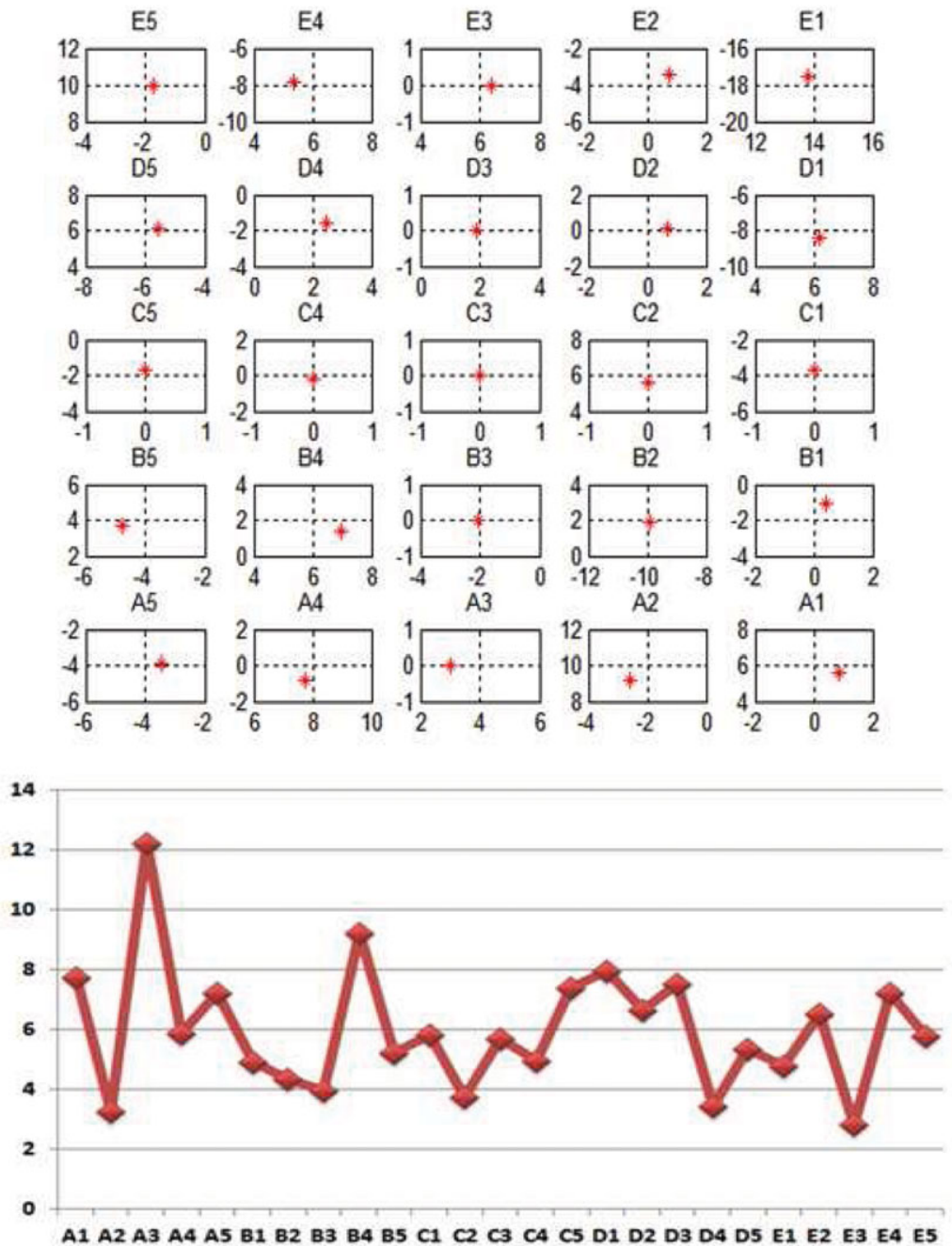

Figure 3. Position error (top) \& aperture error (bottom) of 25 drilling holes

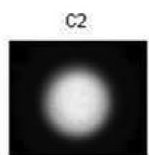

$\mathrm{D} 2$

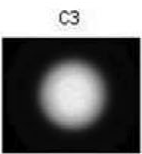

D3

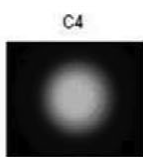

D4
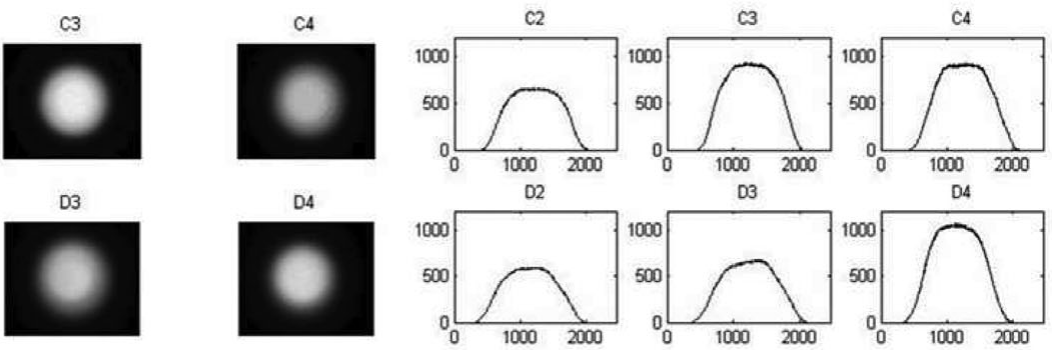

Figure 4. Part of Spot photo (left) and flux curve (right) 

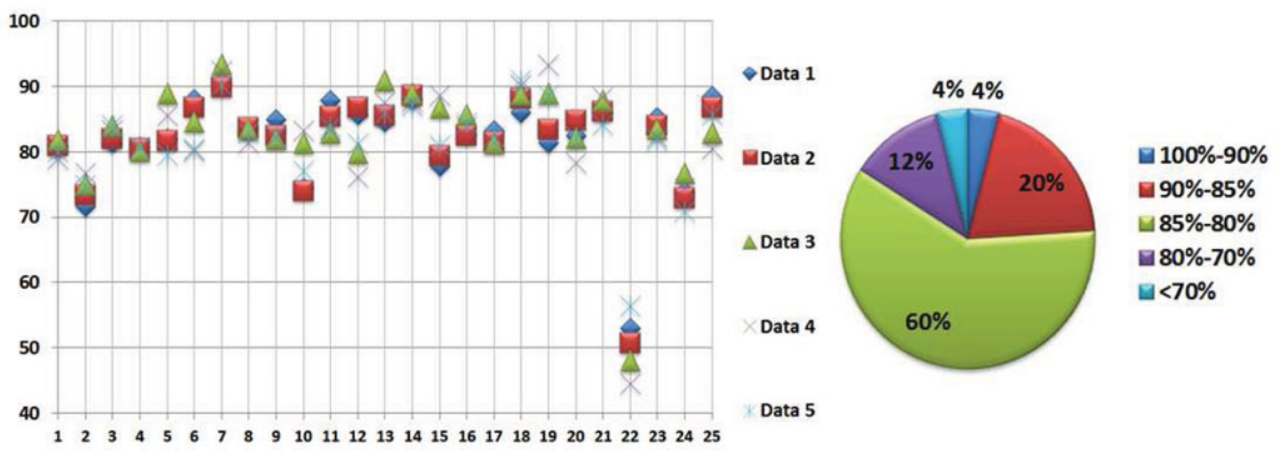

Figure 5. Coupling Efficiency

steps, including higher-precision casting means, the concentricity of ceramic ferrules and the design optimization.

This research received funding from Joint Fund of Astronomy/ NSFC and CAS (No. 10978004), and Young Science Fund / NSFC (No. 11103051).

\section{Reference}

Kai, Zhang, Yongtian, Zhu, Lei, Wang, Zhongyu, Yue, Yi, Chen, Jin, Tang \& Zhongwen, Hu, 2012, Proc. SPIE, 8446, 312 